\title{
Computational Materials Science at the Cutting Edge
}

\author{
Stefan Blügel \\ Institut für Festkörperforschung, Forschungszentrum Jülich, \\ 52425 Jülich, Germany \\ s.bluegel@fz-juelich.de \\ Phone: +49 246161 4249; Fax: +492461 612850 \\ www.fz-juelich.de/iff/staff/Bluegel_S/
}

\begin{abstract}
Material science is a field which cuts through physics, chemistry, biology, and engineering working with an enormous spectrum of different material systems and structures on quite different length and time scale and symmetry, on different degrees of hardness or softness as well as on liquids. The degree to which new functionalities of magnetic clusters, quantum dots, bio-molecules or carbon nanowires can be exploited for specific applications depends heavily on our ability to design devices with optimal behavior in response to external stimulation, such as applied voltage. While the basic physical effects are often well understood, quantitative simulations with predictive power that do not rely on empirical models and parameters pose a major challenge. This is due to the large numerical effort of the calculations, to accurately describe quantum effects at atomic and larger distances. Therefore, modern quantum simulations in material science depend heavily on efficient algorithms and powerful computer hardware.
\end{abstract}

During the past ten years, first-principles calculations based on the densityfunctional theory (DFT) emerged as the most powerful framework to respond to the demands mentioned above on a microscopic level. By first-principles is meant, that the parameters of the theory are fixed by the basic assumptions and equations of quantum mechanics. The overwhelming success of the densityfunctional theory for the description of the ground-state properties of large material classes including organic molecules and bio-molecules, insulators, semiconductors, semimetals, half-metals, simple metals, transition-metals and rareearths in bulk, at surfaces and as nanostructures such as fullerenes and nanotubes makes it the unchallenged foundation of any modern electronic structure based materials.

In this talk I will explore the opportunities of petaflop computing for materials science. Petaflop computing opens the path for the treatment of the van der Waals interaction of molecules, the chemical reactions of bio-molecules and the treatment of strongly-correlated electrons, where concept of individual electrons breaks down. These problems benefit from the advent of massively parallelized computers. Conclusions for the method development for massively parallelized computers are drawn. 